\title{
KCC3 axonopathy: neuropathological features in the central and peripheral nervous system
}

\author{
Roland N Auer ${ }^{1}$, Janet L Laganière ${ }^{2}$, Yves O Robitaille ${ }^{3}$, John Richardson ${ }^{2}$, Patrick A Dion ${ }^{2}$, \\ Guy A Rouleau ${ }^{2}$ and Masoud Shekarabi ${ }^{4}$
${ }^{1}$ Department of Pathology, University of Saskatchewan, Saskatoon, Saskatchewan, Canada; ${ }^{2}$ Department of Neurology and Neurosurgery, Montreal Neurological Institute and Hospital, McGill University, Montréal, Québec, Canada: ${ }^{3}$ Department of Pathology, Hôpital Ste-Justine, Faculty of Medicine, University of Montréal, Montréal, Québec, Canada and ${ }^{4}$ Department of Neuroscience, Lewis Katz School of Medicine at Temple University, Philadelphia, PA, USA

\begin{abstract}
Hereditary motor and sensory neuropathy associated with agenesis of the corpus callosum (HMSN/ACC) is an autosomal recessive disease of the central and peripheral nervous system that presents as early-onset polyneuropathy. Patients are hypotonic and areflexic from birth, with abnormal facial features and atrophic muscles. Progressive peripheral neuropathy eventually confines them to a wheelchair in the second decade of life, and death occurs by the fourth decade. We here define the neuropathologic features of the disease in autopsy tissues from eight cases. Both developmental and neurodegenerative features were found. Hypoplasia or absence of the major telencephalic commissures and a hypoplasia of corticospinal tracts to half the normal size, were the major neurodevelopmental defects we observed. Despite being a neurodegenerative disease, preservation of brain weight and a conspicuous absence of neuronal or glial cell death were signal features of this disease. Small tumor-like overgrowths of axons, termed axonomas, were found in the central and peripheral nervous system, indicating attempted axonal regeneration. We conclude that the neurodegenerative deficits in HMSN/ACC are primarily caused by an axonopathy superimposed upon abnormal development, affecting peripheral but also central nervous system axons, all ultimately because of a genetic defect in the axonal cotransporter KCC3.
\end{abstract}

Modern Pathology (2016) 29, 962-976; doi:10.1038/modpathol.2016.90; published online 27 May 2016

Hereditary motor and sensory neuropathy associated with agenesis of the corpus callosum (HMSN/ACC, Online Mendelian Inheritance in Man \#218000), also known as ACC associated with peripheral neuropathy (ACCPN) or Andermann syndrome is a rare autosomal recessive disorder that occurs in focal geographic regions with founder effects or where inbreeding is prevalent. It was first described in the Charlevoix region of Québec, Canada. ${ }^{1}$ HMSN/ACC has both developmental and neurodegenerative features.

Signs of HMSN/ACC appear early; the patients are hypotonic and hyporeflexic from birth. Parents often notice hypotonia during the first year of life with subsequent delays in motor milestones. Progressive sensorimotor polyneuropathy leads to areflexia by age 2-3 years. Clinical features also include dysmorphic facies, hypotonia and cognitive

Correspondence: Dr M Shekarabi, MSc, PhD, Department of Neuroscience, Lewis Katz School of Medicine at Temple University, 3500N Broad Street, Philadelphia, PA 19140, USA.

E-mail: masoud.shekarabi@temple.edu

Received 19 January 2016; revised 4 April 2016; accepted 5 April 2016; published online 27 May 2016 impairment. During childhood, motor functions develop slowly, with some patients unable to stand or walk without support by $4-6$ years of age. ${ }^{1}$ This stage is followed by progressive motor deterioration, often with development of scoliosis, such that the majority of subjects are wheelchair bound by 12-15 years of age. Most HMSN/ACC patients develop speech but are severely dysarthric. Convulsions are frequent and many patients develop neuropsychiatric syndromes, with visual and auditory hallucinations, paranoid delusions, and a depressive state. ${ }^{2}$

ACC has high penetrance in HMSN/ACC patients. Mathieu et $a l^{3}$ studied a group of 64 patients and found $57.8 \%$ with complete, $9.4 \%$ with partial and $32.8 \%$ with no radiological signs of ACC. Neither the significance nor the causes of the variable involvement of the corpus callosum are known, but it is not correlated to the natural course of the disease and the neurodegenerative features of HMSN/ACC, which do not vary significantly between individuals with or without ACC. ${ }^{3}$ HMSN/ACC patients die in their late 20 to 30 s because of respiratory failure. 
Previous neuropathological observations in HMSN/ACC patients have included the presence of small oval vacuoles in the brain white matter, signs of axonal swelling and depletion of large myelinated fibers in nerves. ${ }^{4}$ Pathological findings from muscle and nerve biopsies, reported by Larbrisseau et $a l^{5}$, revealed evidence of chronic denervation with angular fibers and type grouping, suggesting an axonal neuropathy and a major primary defect at the level of peripheral nerve in HMSN/ACC. ${ }^{5}$

In French-Canadian HMSN/ACC patients, a mutation was identified in a gene, which encodes a potassium/chloride cotransporter (KCC3), solute carrier family 12 member 6 (termed SLC12A6) located on chromosome 15q13-q14. The most common mutation was found to be a deletion of guanine in exon 18 at position 2436, which affects KCC3 RNA splicing leading to the formation of a premature stop codon at amino-acid 813. ${ }^{6}$ Additional KCC3 mutations were also found in HMSN/ACC patients. ${ }^{4,7,8}$ KCC3 belongs to a small family of electroneutral cotransporters and has been implicated in cell volume control by extrusion of potassium and chloride ions along with water to the extracellular environment. ${ }^{9}$

The observation of a watery swelling of axons and of spheroids has been established using histochemical methods. ${ }^{10}$ However, there has been no comprehensive analysis of cellular pathogenesis of HMSN/ ACC nervous tissues using immunodetection methods and cellular markers. Here, we used these methods to analyze all brain regions in nervous tissue derived from eight autopsies of French-Canadian HMSN/ACC patients with the exon 18 mutation. Our results indicate widespread changes in the entire central and peripheral nervous system. We have established neurodevelopmental deficits in major telencephalic commissures, as well as in corticospinal tracts, which appeared to be primary and not caused by neurodegeneration events. A sequential pathogenesis was found, with clear axonal swelling, followed by neurofilamentous accumulation and finally mitochondrial flocculent densities (MFDs). The last change is usually associated with neuronal cell death in neurologic disease, but here was limited to the axon. The most severe neurodegenerative features were predominantly found in the peripheral nervous system and motor systems rather than the central nervous system and sensory systems. The widespread axonal degeneration found was not accompanied by neuronal death.

\section{Materials and methods}

Available historical data were obtained from the archives of the Montreal Neurological Institute. Some histories were incomplete. All nervous tissues were obtained with the consent of the parents or caregivers for use in research according to the protocols approved by the ethics committee on human experimentation of the Centre Hospitalier de l'Université de Montréal and McGill University Health Center. Here, we present all archival cases of HMSN/ACC based on published phenotypic and genetic criteria. ${ }^{2,5,6,9}$

There were eight autopsies of patients who died between the ages of 23 and 37 years. Post-mortem tissue was procured from autopsies performed within 24 or $48 \mathrm{~h}$ after death. Tissues were fixed in formalin for light microscopy, and in glutaraldehyde and osmium tetroxide for electron microscopy. Brains were cut and photographed 2-4 weeks after immersion in $10 \%$ buffered formalin. Tissue blocks were prepared, embedded in paraffin and conventionally stained for hematoxylin and eosin, luxol fast blue, Sudan Black B, Bodian and periodic acid-Schiff using standard protocols. By immunohistochemistry, corresponding sections were also stained using an antibody against neurofilamentmedium (Dako; 1:100) or glial fibrillary acidic protein (GFAP) (Sigma; 1:2000) and a secondary biotinylated antibody (anti-mouse immunoglobulin G, Dako; 1:1000) as described previously. ${ }^{11}$ The neurofilament antibody was used to locate axonal spheroids. For high-resolution light microscopy and assessment of sampling for electron microscopy, glutaraldehyde-fixed tissue was embedded in epoxy resin (epon).

Sections were imaged using light microscopy on a Zeiss Axiophot with an AxioCam HRc camera. Regions examined (Figures 1-9) were samples from telencephalon, diencephalon, brainstem, cerebellum and spinal cord and included all major central nervous system nuclei and tracts, as well as peripheral nervous system tissue samples. Electron microscopy and photography was done on a Philips EM $208 \mathrm{~S}$ digital electron microscope.

To quantify the surface areas of the corticospinal tracts at the level of medulla oblongata, the sections were stained with luxol fast blue and scanned into a computer. The pyramids of the medulla were then traced within their anatomical limits as bounded by the pia mater anteriorly, the inferior olive laterally, the medial lemniscus dorsally and the median glial septum of the medulla oblongata mesially. The surface areas were then measured using Image J software as described previously. ${ }^{11}$ To take the level of the cut and possible variations in the medulla's size into consideration, the mean of surface areas of the pyramids on one side (Sp) was divided by the mean of total surface area of the ipsilateral medulla (Sim) to obtain Psr $(P=$ patient and $\mathrm{sr}=$ surface area $)$ as well as for the control (Csr; $\mathrm{C}=$ control). The fold changes in the surface areas of pyramids of the patients were then obtained using the Psr $\times 1 / \mathrm{Csr}$ equation. 

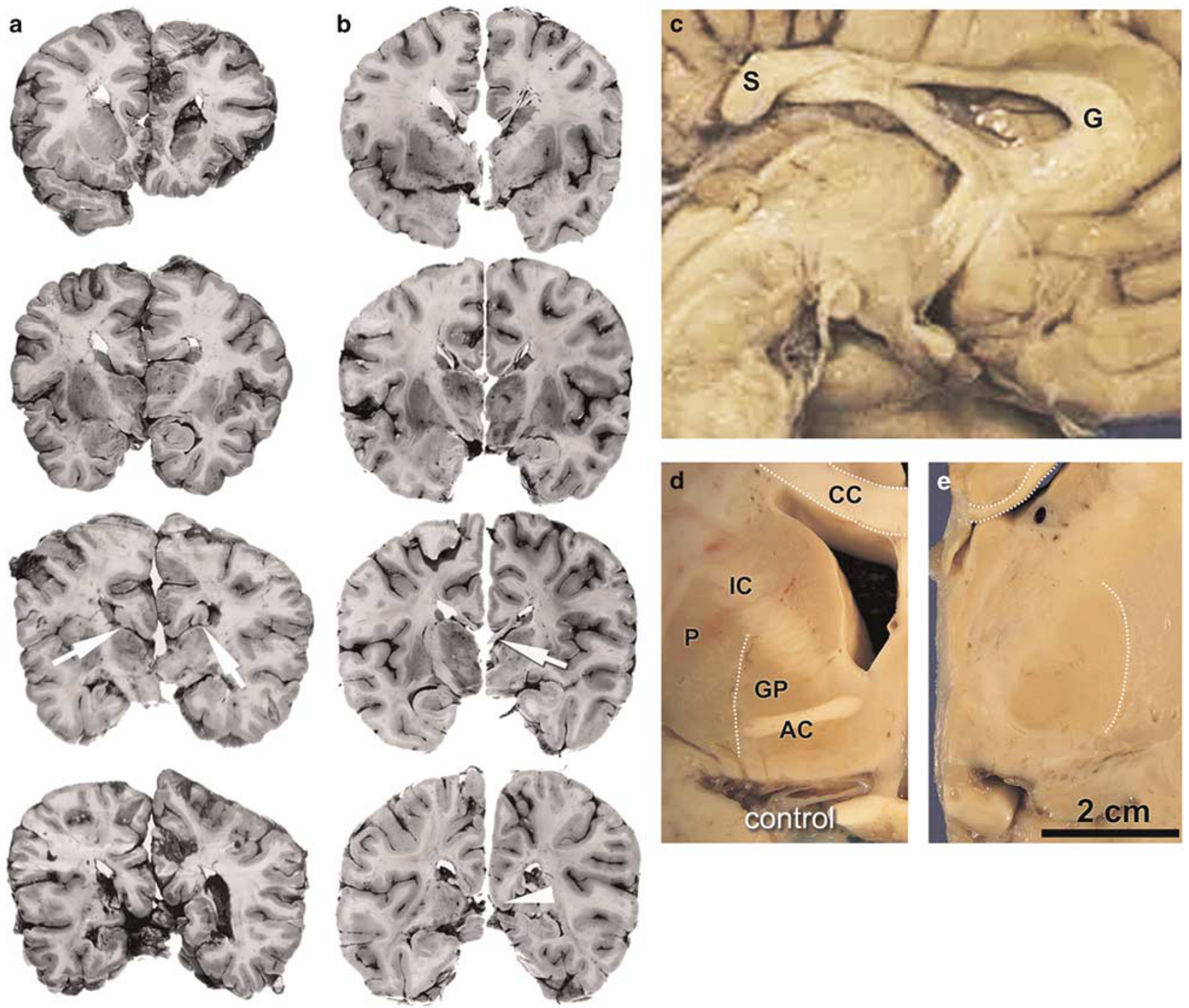

Figure 1 Gross brain examination of HMSN/ACC patients revealed variable degrees of commissure hypoplasia. Vertical montages from two cases with HMSN/ACC are shown on the left. (a) Four coronal slices from case 2 with complete ACC and (b) from case 4, with hypoplasia of the corpus callosum. Overall cortical and gyral structures were normal and brain weights were not reduced. Large, conspicuous bundles of Probst are seen bilaterally in the brain with no corpus callosum (arrows in a). A thin body of the corpus callosum is sometimes present in this disease (arrow in b), with the splenium missing (arrowhead in b). (c) Mesial view of a telencephalon (case 6) with a complete corpus callosum from genu (G) to splenium (S), but hypoplastic throughout, and concomitant complete aplasia of the anterior commissure. (d) Coronal slices at the level of the optic chiasm show a control brain with the corpus callosum (CC), anterior commissure (AC), globus pallidus (GP), putamen (P) and internal capsule (IC). (e) The dashed white lines allow size comparison of several brain structures within the diseased brain (case 6).

\section{Results}

\section{Macroscopic Description}

Brain and body weights were noteworthy. All cases had extremely low body weights and heights, except case 6, who developed obesity (Supplementary Materials, Table 1). Strikingly, the average weight of the six brains exceeded the average weight of a normal brain. The brain weights of six of the cases were 1524, 1460, 1400, 1440, 1650 and $1450 \mathrm{~g}$ (mean of $1487 \mathrm{~g}$ ), respectively. The neocortex appeared generally normal in all cases, without atrophy. Case
3 showed an old cavitated infarct at the base of the right temporal and occipital lobes with no unusual histological features. In cases 1, 6 and 8, the hippocampus was slightly rotated laterally.

Commissural abnormalities were a major feature. In cases 1,2 and 3, the corpus callosum was entirely absent (Figure 1a). In case 3, a small rounded portion of the corpus callosum was present anteriorly, where a thin lamina terminalis connected it to the anterior commissure. There were prominent bundles of Probst attached to the fornices, whereas the gyral pattern on the medial side of the hemispheres corresponded to the radial pattern generally seen 


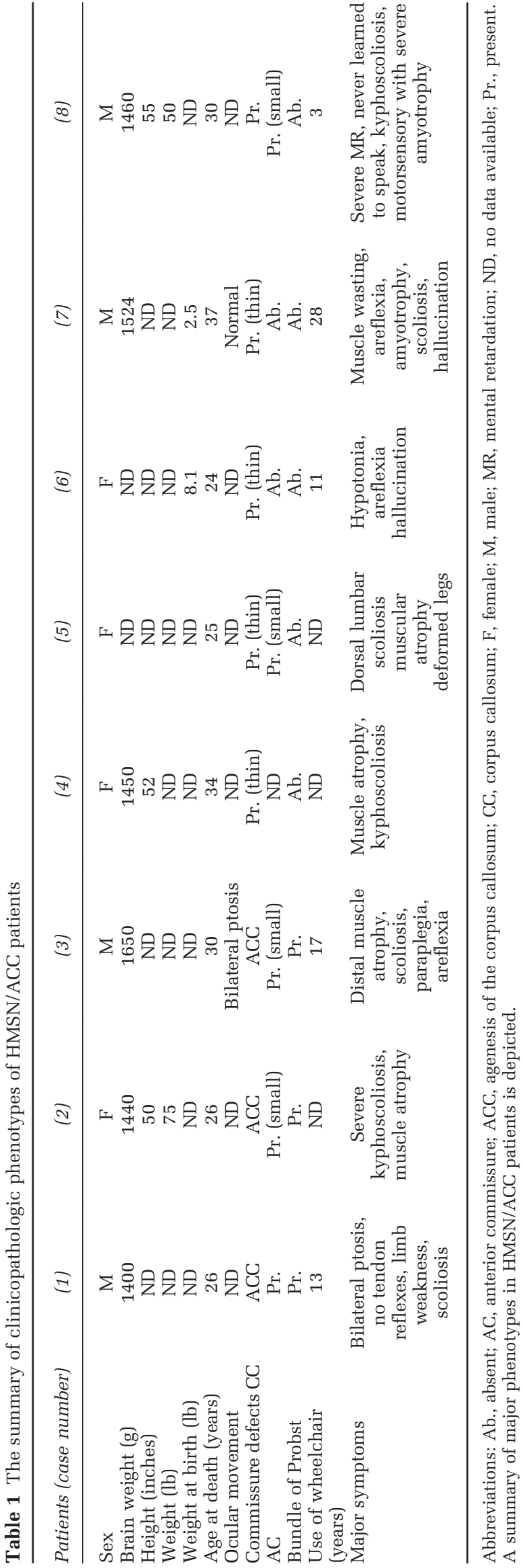

with callosal agenesis (Figure 1a, arrows). In cases 4, 5 and 6 , the corpus callosum was thin and its anteroposterior length was short (Figure $1 \mathrm{~b}$, arrows and Figure 1c). In cases 7 and 8, the corpus callosum was present in its entirety, although the posterior portion was thinner than the anterior portion. The anterior commissure was completely absent in cases 6 and 7 (Figures 1c-e), and attenuated in cases 2, 3 and 8.

Corticospinal tracts were small and explained clinical motor features of the disease. Hypoplasia of the pyramids in the medulla oblongata was observed in four cases (1, 2, 5 and 6) (Figures 2a-e). Planimetric measurements of the ratios of the pyramids to the remaining ipsilateral medulla revealed a $\sim 50 \%$ reduction in the pyramids versus control (Figure 2f). The clinical history of all four cases in the extent of their ability to walk corresponded to the size of their pyramids. For example, case 6 never walked and had the smallest pyramid, and case 5 was confined to a wheelchair at age 28 and appeared to have a larger pyramid.

There was no correlation between the extent of axonal swelling or degeneration along the corticospinal tracts in superior areas and the size of the pyramid. In fact, in case 8 , who had the smallest pyramid, the corticospinal tracts in the cerebral peduncle of the midbrain displayed the same axonal density pattern as seen in the other patients, possibly indicating a guidance error in the corticospinal projections rather than simply neurodegeneration. A few spheroids stained as empty vacuoles on myelin stain or as immunopositive neurofilamentfilled spheroids, within the corticospinal tracts (Figures $2 \mathrm{~g}$ and $\mathrm{h}$ ).

The spinal cord, examined in cases 1, 5, 6 and 7, was macroscopically normal, although in case 3 some of the roots of the cauda equina appeared slightly enlarged.

\section{Microscopic Findings}

In the brain white matter of all cases, hematoxylineosin staining showed pale pink, but occasionally virtually unstained scattered oval vacuoles or spheroids, either empty or containing a small eosinophilic web. These were negative with the periodic acidSchiff stain.

The corpus callosum of cases 5, 7 and 8 appeared microscopically normal in its anterior portion except for infrequent vacuoles. There was progressively more evidence of axonal loss proceeding posteriorly, with tissue atrophy causing increased packing density of oligodendroglial cells and proliferation of astrocytes, which were also observed in GFAP staining of pons and cortex (Supplementary Figure S1) There was no evidence of microglial activation in sections stained with antibody against cluster of differentiation 68 (CD68) (data not shown). Occasional pinkish, slightly enlarged axons could be 


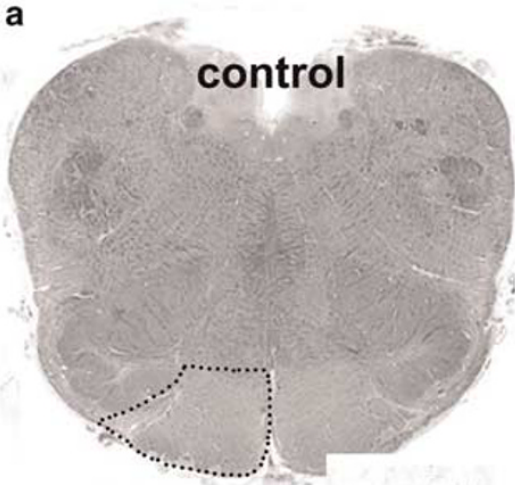

d

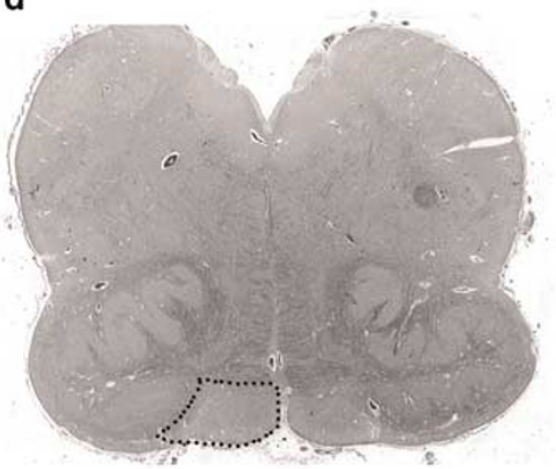

b

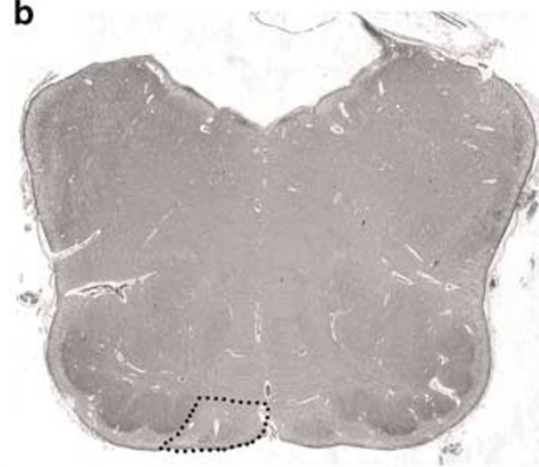

e

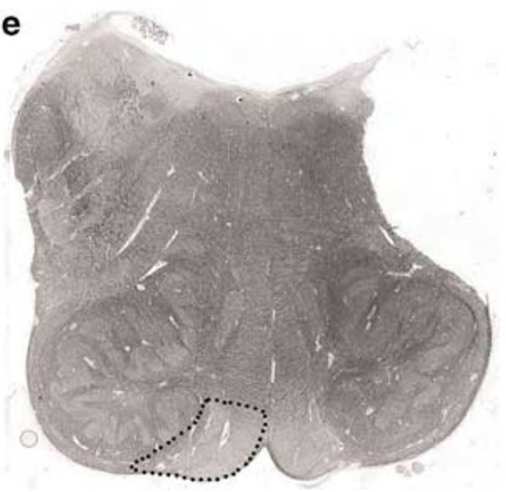

C

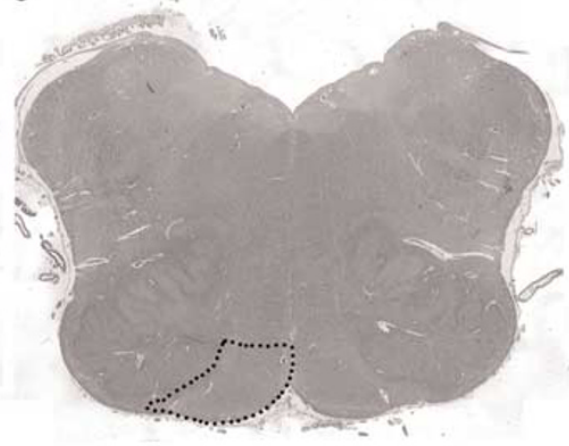

f
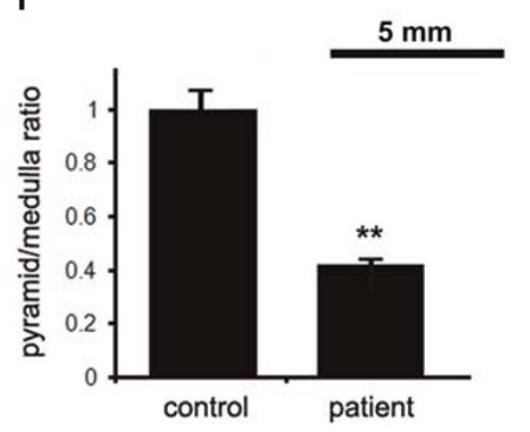
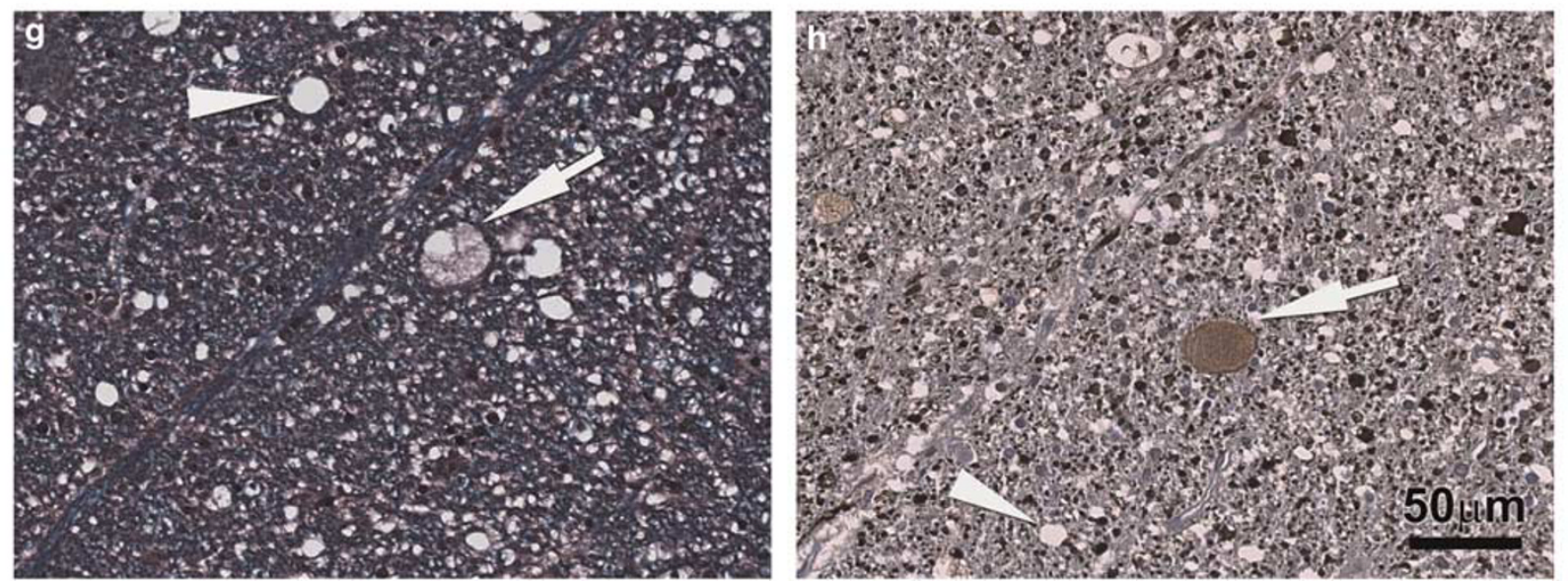

Figure 2 A major developmental deficit was observed in the corticospinal tracts of the pyramids. Corticospinal tract hypoplasia is seen in the medulla oblongata of the patient versus the control (a). The pyramids were small in all cases, but to a variable degree. (b-e, cases $8,7,3$ and 4, respectively). (f) Planimetric ratios of the pyramidal areas (dotted) over total cross-sectional areas of the ipsilateral medulla showed the pyramids on average to be half the normal size. Compared with this developmental abnormality, evidence of active degeneration was meager: histology of the pyramids showed a few spheroids within the myelin sheath (g, case 3), and using an antibody against neurofilament protein (h, case 7). Neuraxonal spheroids were either filled with neurofilament-containing axoplasm (arrows in $\mathbf{g}$ and $\mathbf{h}$ ) or were clear and empty (arrowheads in $\mathbf{g}$ and $\mathbf{h}$ ). ${ }^{* *} P<0.05$. control: $n=2$, patients: $n=4, t$-test).

seen using the hematoxylin-eosin stain, but where axonal loss was greatest, large vacuoles were absent.

In all cases with a corpus callosum, the posterior part displayed significantly more spheroids than the rest of the corpus callosum when stained with a neurofilament marker (Figures 3a-c). Few spheroids were found in the posterior and anterior commissures (Figures 3d and e and Table 2). In case 3, the small callosal remnant was histologically normal, but there was evidence of axonal loss in the bundle of Probst. In fact, neurofilament staining showed some spheroids within the bundle of Probst (Figure 3f), indicating an overlay of neurodegeneration on abnormal development. In cases 4 and 7, slight demyelination of the corpus callosum was observed in the anterior portion of the splenium using luxol fast blue staining.

Among the brain white matter tracts, the increase in axon diameter was apparent and more marked in 

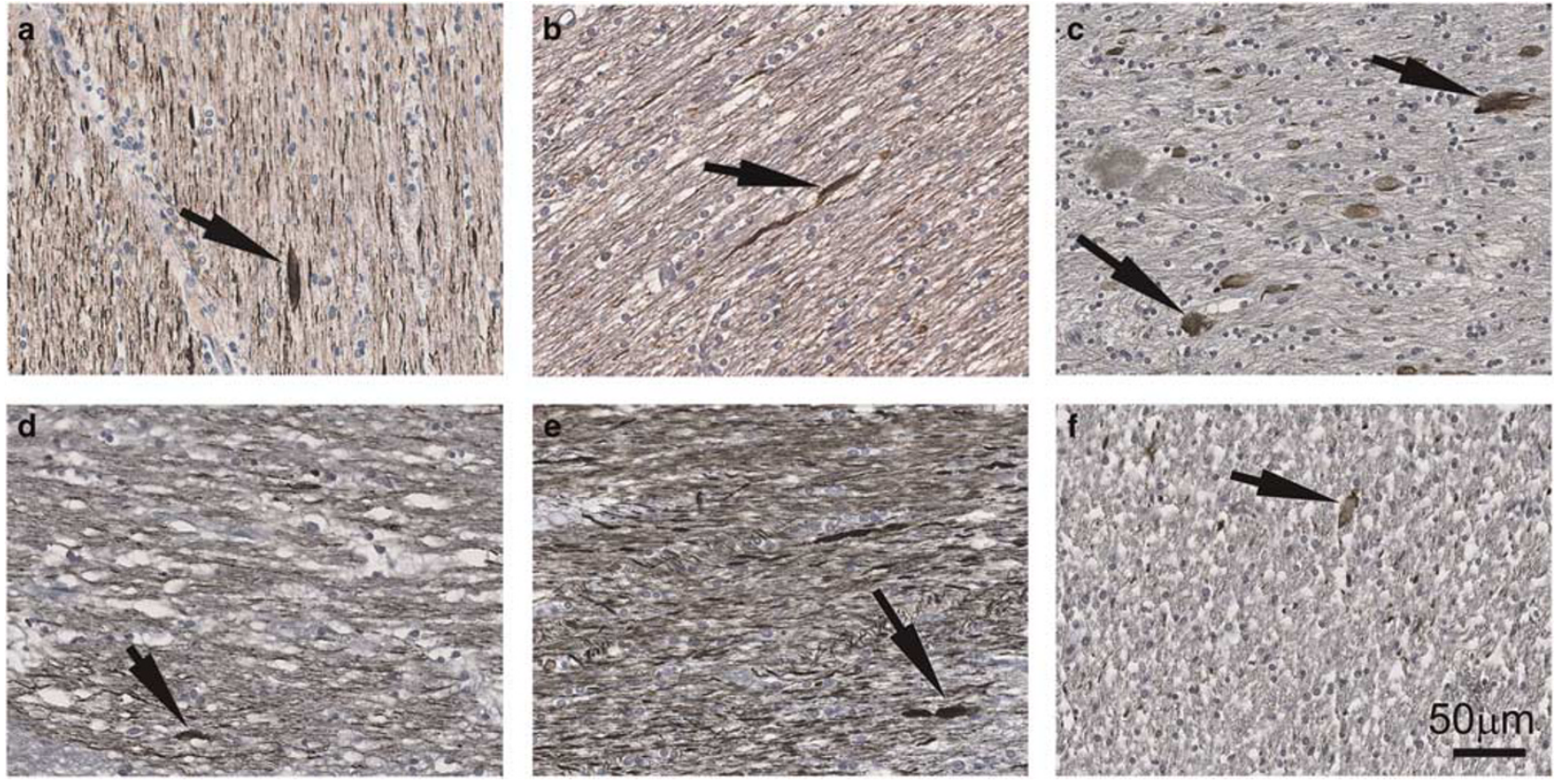

Figure 3 Neuraxonal spheroids were not prominent in the major commissures of the brain. The corpus callosum showed spheroids (arrows) in (a, case 5) the genu, (b, case 5) body and (c, case 7) splenium, as seen here in longitudinal sections. The splenium displayed more spheroids than other areas within the corpus callosum. Spheroids (arrows) were also present in the posterior commissures (d, case 8), anterior commissures (e, case 5) and in the bundles of Probst (f, case 3), the aberrant fibers running in an antero-posterior direction rather than forming the corpus callosum. All the sections were immunostained with an antibody against neurofilament protein.

the brainstem than the brain, being particularly prominent in the corticospinal tracts, decussating axons in the midbrain, pons and medulla, the decussation of the brachium conjunctivum and the medullary internal arcuate fibers, medial and lateral lemniscus. In contrast, axonal spheroids were much less apparent in medial longitudinal fasciculus (MLF) (Figures 4a-l and Table 2).

Fewer spheroids were found in telencephalic areas than brainstem. However, the centrum semiovale displayed spheroids, which were lightly stained or appeared negative as watery vacuoles (Figures 5a and b). Occasional axonal dilatations were observed in the basal ganglia (Figures 5c and d and Table 2). These spheroids were often intensely stained with a neurofilament protein antibody. In the left cerebral peduncle of case 3, there was a large collection of contiguous pale enlarged axons. More distally in the peduncle, this tract appeared normal.

Compared with the central, peripheral nervous system showed more striking pathology, particularly in the nerve roots where sections of the intradural portion of the third cranial nerve showed loss of normal structure, with marked loss of axons and endoneurial fibrosis. Remaining axons showed varying degrees of dilatation up to approximately $120 \mu \mathrm{m}$ in diameter. Using hematoxylin and eosin, these usually stained pale pink, but they were occasionally virtually unstained. With the Bodian stain, the contents were pale gray. The periphery of the spheroids was usually lined by Schwann cell nuclei. With Sudan Black B, no myelin could be stained around the most enlarged axons, whereas moderately enlarged axons showed thin sheaths with luxol fast blue staining (Figure 5g). Remnants of axoplasm occupied variable proportions of these dilated axons. Some axons showed little or no remnants of axoplasm, mostly clear spaces (Figure 5e). The neurofilament antibody stained some, where they showed axoplasm with clear content of neurofilaments, but not others (Figures $5 \mathrm{f}$ and h, arrows). The antibody also detected axoplasmic debris, which occasionally filled dilated axons (Figure 5h, arrowhead).

In sensory nerve rootlets, moderate axonal loss was noted, which displayed the same spectrum of changes as in motor rootlets, but greatly reduced in severity. Dilated axons were much less frequent and the packing density of myelinated fibers was near normal in sensory nerves (Figures 6a-c). However, near the cord, pure motor rootlets were much more affected than sensory ones. Mixed nerves fell somewhere in between. Significant signs of axonal abnormalities were seen in the third cranial nerve (Figures 6d-f).

In addition to axons, we examined the integrity of neuronal perikarya in different areas of the nervous system. Neurons of the dorsal root sensory ganglia, anterior horns, cortex, as well as neurons in various brainstem nuclei, all appeared normal. However, 
Table 2 A semiquantification of observed pathology in the nervous system of HMSN/ACC patients

\begin{tabular}{|c|c|c|c|c|c|}
\hline Area & Score & Area & Score & Area & Score \\
\hline Cerebral cortex & & Reticular formation & + & Medial lemniscus & + \\
\hline Frontal & + & Oculomotor nucleus & - & Inferior olive, amiculum & + \\
\hline Temporal & + & Cuneiformis nucleus & + & Spinal trigeminal tract & + \\
\hline Parietal cortex & + & Trochlear nucleus & - & Tractus solitarius & + \\
\hline Occipital cortex & + & Mesencephalic nucleus & - & Nucleus ambiguus & - \\
\hline Centrum semiovale & ++ & Superior colliculus & + & Accessory olivary nuclei & - \\
\hline Cingulate gyrus & - & Inferior colliculus & + & Inferior olivary nucleus & - \\
\hline Hippocampus & + & Medial longitudinal fasciculus & + & Spinal cord & \\
\hline Fornix & ++ & Superior cerebellar peduncle & ++ & Posterior horn & + \\
\hline Basal ganglia & & Pons & & Lateral horn & + \\
\hline Putamen & + & Locus coeruleus & + & Anterior horn & + \\
\hline Globus pallidus & + & Corticospinal tracts in basis pontis & ++ & PNS & \\
\hline Amygdaloid & + & Vestibular nuclei & & Sensory & \\
\hline Thalamus & + & Pontine nuclei & + & Olfactory nerve (I) & - \\
\hline Cerebellum & & Middle cerebellar peduncle & + & Optic nerve (II) & + \\
\hline Purkinje and granule & & & & & \\
\hline cell layers (axons) & + & Central tegmental tract & ++ & Sensory trigeminal (V) & ++ \\
\hline Dentate nucleus & + & Facial nerve & ++ & Vestibulocochlear neve (VIII) & + \\
\hline Pulvinar & + & Medulla oblongata & & Spinal sensory rootlet & + \\
\hline Corpus callosum & & Vestibular nuclei & - & Motor & \\
\hline Splenium & ++ & Nucleus cuneatus & - & Oculomotor (III) & ++++ \\
\hline Body & + & Nucleus gracilis & - & Spinal motor rootlet & ++++ \\
\hline Genu & + & Hypoglossal nucleus & - & Mixed nerves & \\
\hline Midbrain & & Dorsal nucleus of vagus & - & Facial nerve (VII) & +++ \\
\hline Substantia nigra & - & Olivocerebellar transverse fibers & ++ & Sciatic nerve & ++ \\
\hline Central gray & - & Corticospinal tracts in pyramids & + & & \\
\hline Raphe dorsalis nucleus & - & Reticular formation & + & & \\
\hline
\end{tabular}

Abbreviation: CST, corticospinal tract.

A semiquantification analysis of spheroids dispersion was performed on all examined nervous tissues of the HMSN/ACC patients. Score: $-=$ not observed; += very disperse; ++= moderate; +++= strong; ++++= very severe.

perisomatic axonal spheroids were found surrounding the central nervous system nuclei throughout the central nervous system and in peripheral ganglia (Figures 7a-l). Occasionally, some shrinkage of the neuronal somata was observed, perhaps a result of the dying back of axons. There was, however, a conspicuous absence of neuronal death in our study.

Axonomas or regenerative clusters, ${ }^{12}$ an indication of ongoing degeneration and an aberrant tumor-like regenerative axon cluster, were more frequently observed in the peripheral than central nervous system as thinly myelinated axons growing in different directions or multiple axons confined within a thin single myelin sheath (Figures 8a-d). Axonomas were not seen in any sensory roots. Their presence was noticed in the dorsal horn of the spinal cord of case 7 (Figure 8e). Small myelinated fibers were often clustered.

Epoxy resin sections of roots confirmed the findings on paraffin sections, also showing moderate axonal loss. Most myelin sheaths were uniform in thickness around the periphery of the axons. Some axons had no myelin detectable by light microscopy, but there was no evidence of active myelin degradation or digestion by macrophages. Infrequently seen in peripheral nerve were cytoplasmic residues, which still were attached to the myelin, with some myelin disintegration detectable (Figures 9a-c). Dispersed neurofilament protein and flocculent densities filled the axoplasm in other locations (Figure 9a).

MFDs were frequent when axons were examined by electron microscopy (Figure 9d) and preceded axonal cell membrane breakdown. Most of the remaining myelin, although thin, was still densely compacted. The peripheral nerve myelination was without abnormality at the electron microscopy level (Figure 9e), including perfectly formed SchmidtLantermann clefts (Figure 9f).

\section{Discussion}

The clinical characteristics of HMSN/ACC, together with our neuropathological findings presented here, indicate that KCC3 expression is important for both normal development and is also important physiologically to sustain axons into postnatal life. The very small body weights are likely accounted for by the lack of general trophic influence of the motor output of the brain on the body size, during both development and degeneration in this disease. The selective axon-killing feature, which preserved neuronal perikarya and other brain cells, thereby preserves brain weight and, together with the somatic stunting, gives rise to a substantial brain 

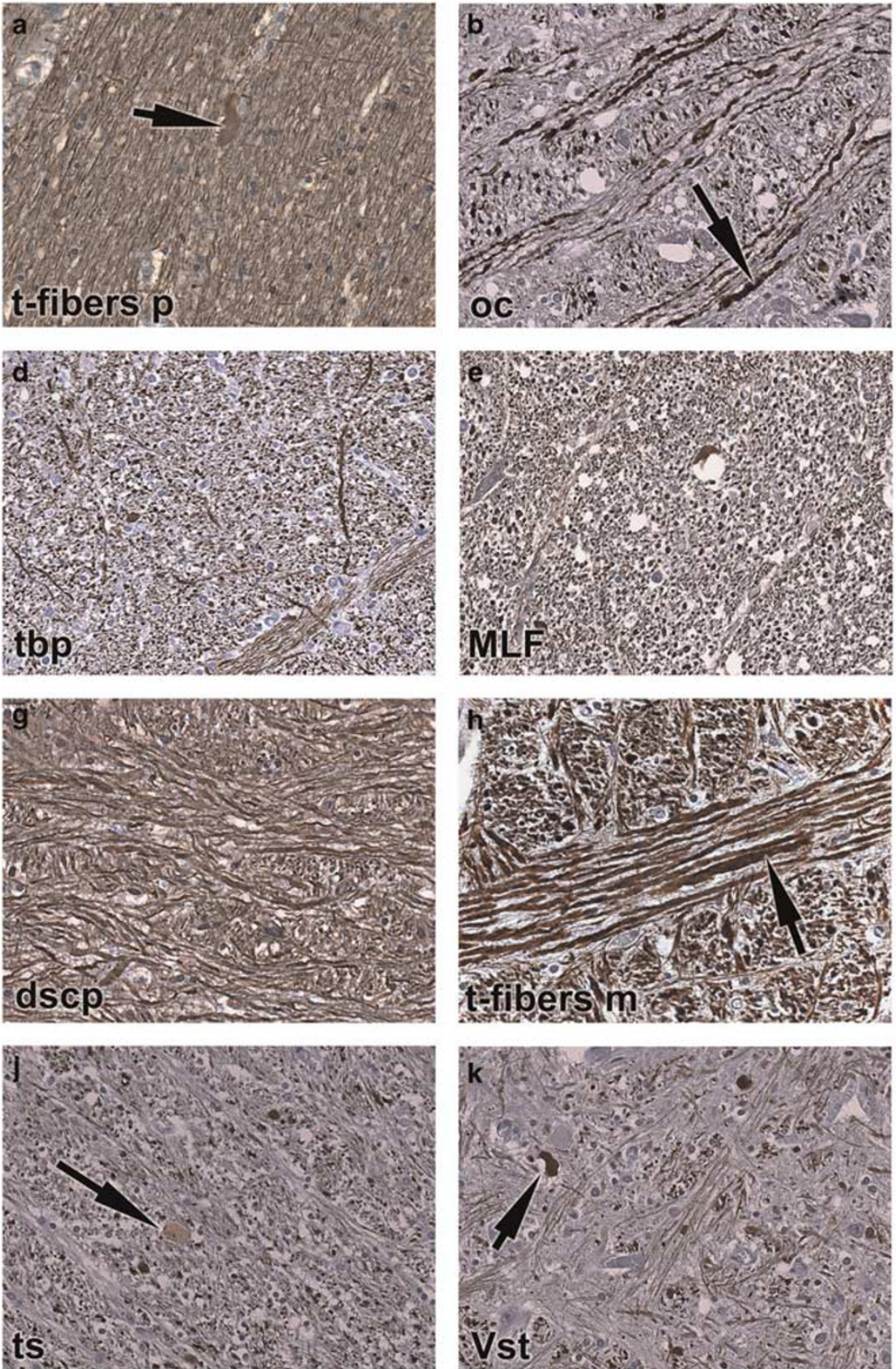
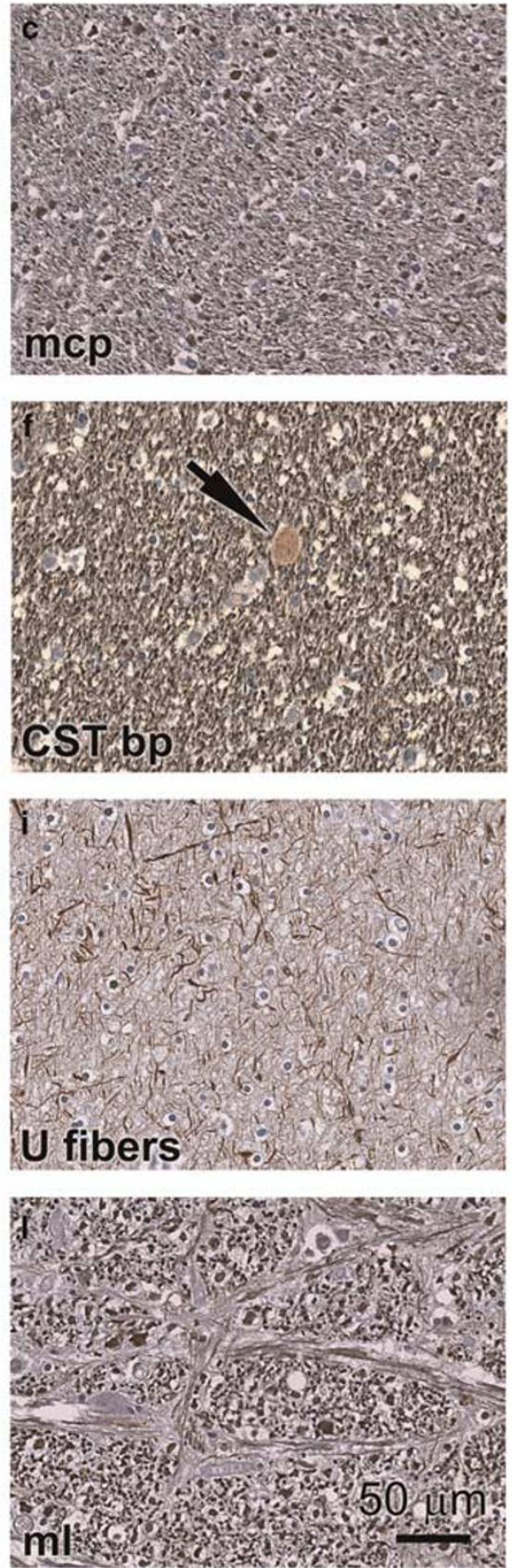

Figure 4 The spheroids were sporadically found in central nervous system white matter outside of the commissures when the brain sections were immunostained against neurofilament protein. In HMSN/ACC patients, the transverse fibers of the basis pontis (a, t-fibers p, case 8), decussating olivo-cerebellar fibers in the medulla oblongata (b, oc, case 4), middle cerebellar peduncle (c, mcp, case 8), transverse pontine fibers of basis pontis (d, tbp, case 3), medial longitudinal fasciculus (e, MLF, case 4), corticospinal tracts in the basis pontis (f, CSTbp, case 8), decussation of the superior cerebellar peduncle (g, dscp, case 8), transverse fibers in the medial lemniscus (h, t-fibers $\mathrm{m}$, case 8), subcortical U fibers in neocortex (i, U fibers, case 8), tractus solitarius ( $\mathbf{j}$, ts, case 7), trigeminal spinal tract (k, Vst, case 7) and medial lemniscus (l, ml, case 7) all show axonal dilatations of variable size when stained with an antibody against neurofilament. Compared with the surrounding normal axons, positive neurofilament staining is variably higher or lower in different spheroids, seen here as pale staining spheroids in (f) and (j), but dark punctate spheroids in (b) and (k), suggesting high and low neurofilament content above and below the neurofilament staining in normal axons (see also Figure 3).

weight/body weight ratio abnormalities. With respect to development, the KCC3 cotransporter is implicated, directly or indirectly, in giving rise to a defect in axonal guidance. This appears to selectively affect commissural fibers destined to form the anterior commissure and corpus callosum. The finding of a bundle of Probst, the aberrant longitudinal fasciculus remaining from the fibers that would have crossed the midline had a corpus callosum developed, indicates that the defect of the 
corpus callosum in this disease is a true developmental agenesis and is not because of degeneration. The corpus callosum development involves complex stages of the projection of cerebral cortex neurons, axonal extension and subsequent guidance by molecules expressed by midline glial structures. ${ }^{13}$ The variability of commissural defects in the patients is an indication of the key roles of genetic predisposition and other factors in the developing commissures.

The $50 \%$ hypoplasia of the corticospinal tracts of the pyramids in medulla of HMSN/ACC patients suggests a significant disruption in corticospinal tracts. This pathology is seen in L1 syndrome or hydrocephalus with stenosis of the aqueduct of Sylvius where both the corticospinal tracts and the corpus callosum are absent in association with stenosis of the aqueduct of Sylvius. ${ }^{14}$ The malformative component of this disease may also relate to some factor in the corticospinal fluid that damages the axons beginning to cross from one hemisphere to the other in the callosal primordium. It is well known that KCC3 is highly expressed by epithelial cells of the choroid plexus. ${ }^{15}$ Therefore, KCC3 expression may have an important role in the production and biochemical properties of corticospinal fluid.

With respect to degeneration, we show that in this disease, there is selective axonal degeneration. The axonal degeneration spares not only surrounding neurons, glia and neuropil, but also the myelin sheath surrounding diseased axons, suggesting a primary axonal process. Intriguingly, we did not observe any signs of Wallerian degeneration, which is surprising and rather unique in a neurodegenerating disease. We propose a local axonal atrophy where neither the somata nor axons show major pathology, which may lead to neuronal or axonal degeneration. Our results point to the important role of KCC3 in the regulation of the axonal microenvironment. This is supported by our finding the lack of
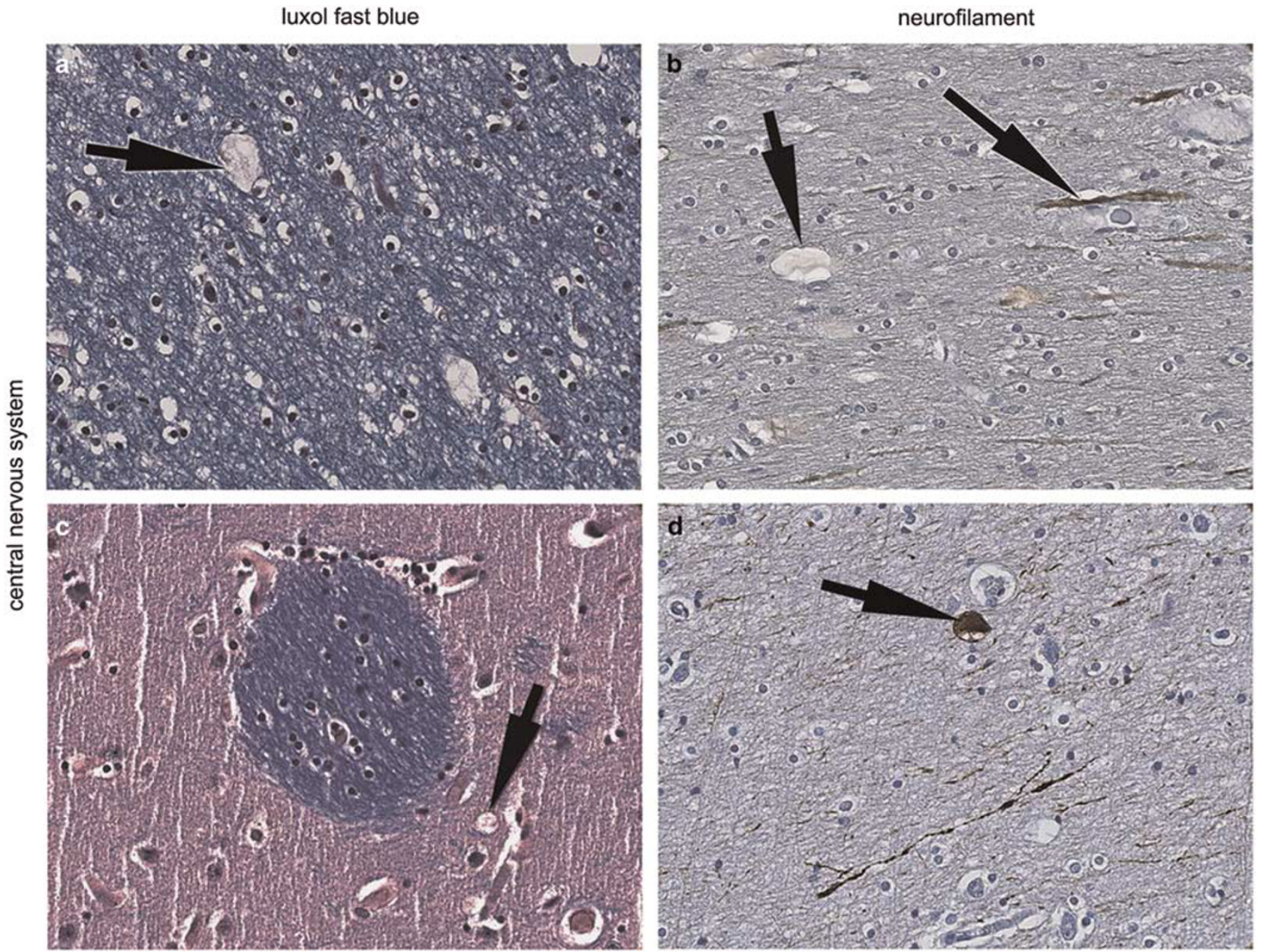

Figure 5 Severe pathology was observed in the peripheral nerves (e-h). The central nervous system (a-d) showed less severe pathology than the peripheral nervous system $(\mathbf{e}-\mathbf{h})$. Panels were stained with luxol fast blue (a, $\mathbf{c}, \mathbf{e}, \mathbf{h})$ or immunostained with an antibody against neurofilament protein (b, d, f, h). The centrum semiovale (a, b, case 4) and the striatum (c, d, case 8) showed a few spheroids with an internal inhomogeneous structure (arrows in a-c). Panels (e, f, case 3) display the facial nerve and (g, h, case 3) show dilated axons in the cauda equina, some with clear fluid surrounding collapsed axoplasm (arrowheads) and others with well-stained axoplasm (arrow in g). Some spheroids were stained with the antibody (arrows in $\mathbf{f}, \mathbf{h}$ ) but other spheroids were empty and vacuole-like (arrowhead in $\mathbf{h}$ ). 

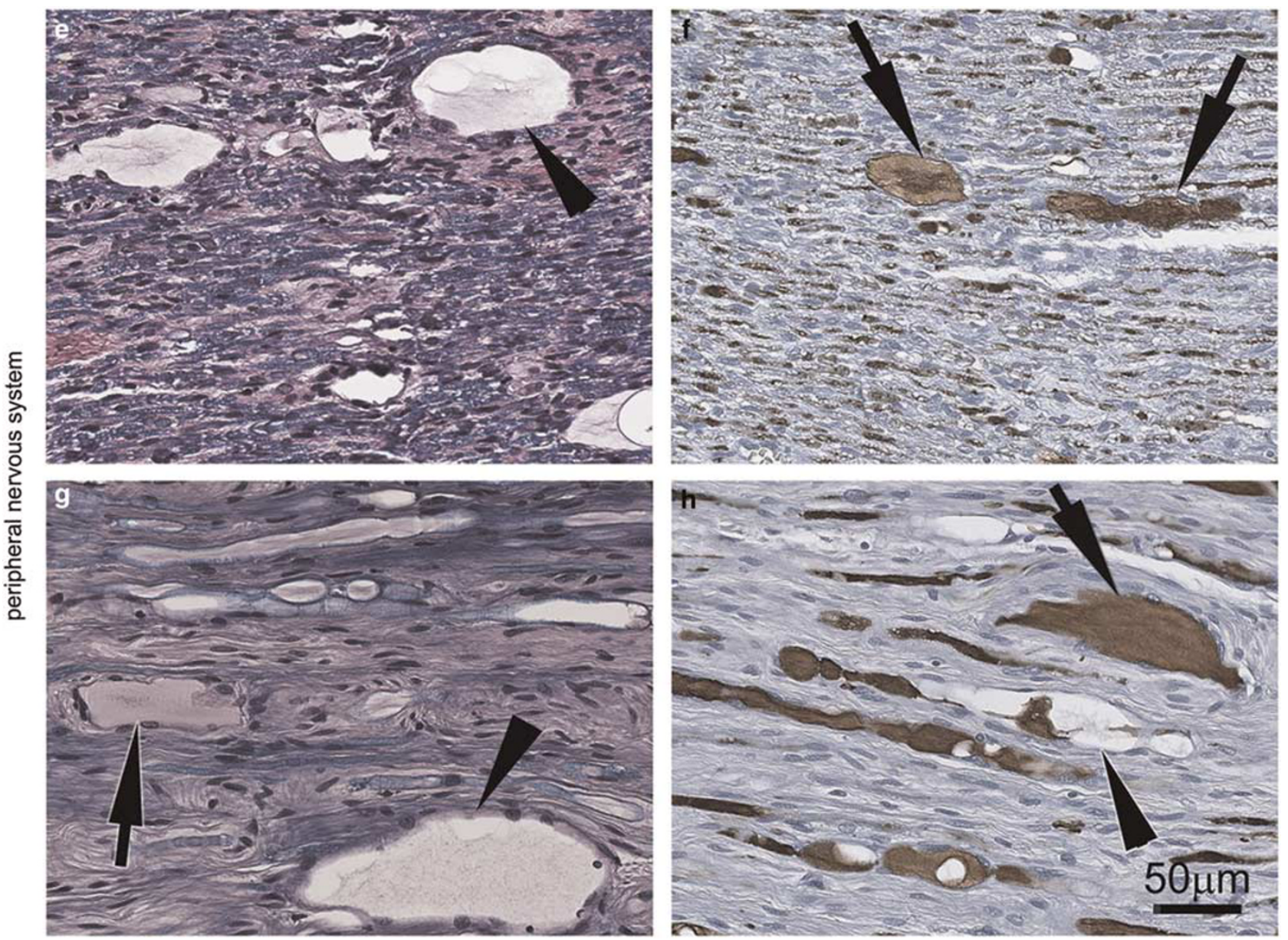

Figure 5 (Continued)

demyelination and the apparent normal myelination even in the most severely affected areas. The specific role of KCC3 in keeping the environment suitable for axons to function properly during development and also during normal axon function is further supported with the observation of collapsed axons in the absence of degeneration or demyelination. The finding of a primarily axonal pathology in HMSN/ ACC patients is in accord with the findings in the animal models. Recently, we observed peripheral neuropathy in a mouse model of HMSN/ACC with a complete selective neuronal knockout of KCC3. ${ }^{11}$ Intriguingly, parvalbumin-specific knockdown of KCC3 has also led to locomotor deficit phenotypes in mice, which supports our previous findings of KCC3 expression in these neurons. ${ }^{16,17}$

Although this disease announces itself as a peripheral neuropathy, our findings of ongoing axonal atrophy in the white matter of the nervous system at the time of death indicate widespread ongoing axonal disease in the cerebral hemispheres as well. Intra-hemispheric disconnection and telencephalic spheroids may explain the psychotic symptomatology in this disease. This is supported by the observation of spheroids present predominantly in the splenium, that carry occipito-parietal and occipital lobe fibers, which are primarily involved in visual processing. This finding also supports the occurrence of visual hallucination in these patients.

The cases in which a corpus callosum was present were the most instructive, as fairly numerous axonal vacuoles were encountered at the beginning of the posterior third of the corpus callosum. Near the splenium of the corpus callosum, vacuoles were almost absent, but the increased packing density of oligodendroglial nuclei suggests considerable old axonal loss and tissue atrophy. This corresponds to the gross appearance of thinness of the posterior part of the corpus callosum. In the central nervous system, aside from the corpus callosum, axonal damage was relatively rare. Within the centrum semiovale, axonal vacuoles were most numerous in the occipital lobes. Our findings of predominantly motor axon neuropathy in HMSN/ACC patients indicates a greater vulnerability of motor versus sensory axons to fluctuations in axonal volume. Although the expression and functional relevance of 


\section{sensory}
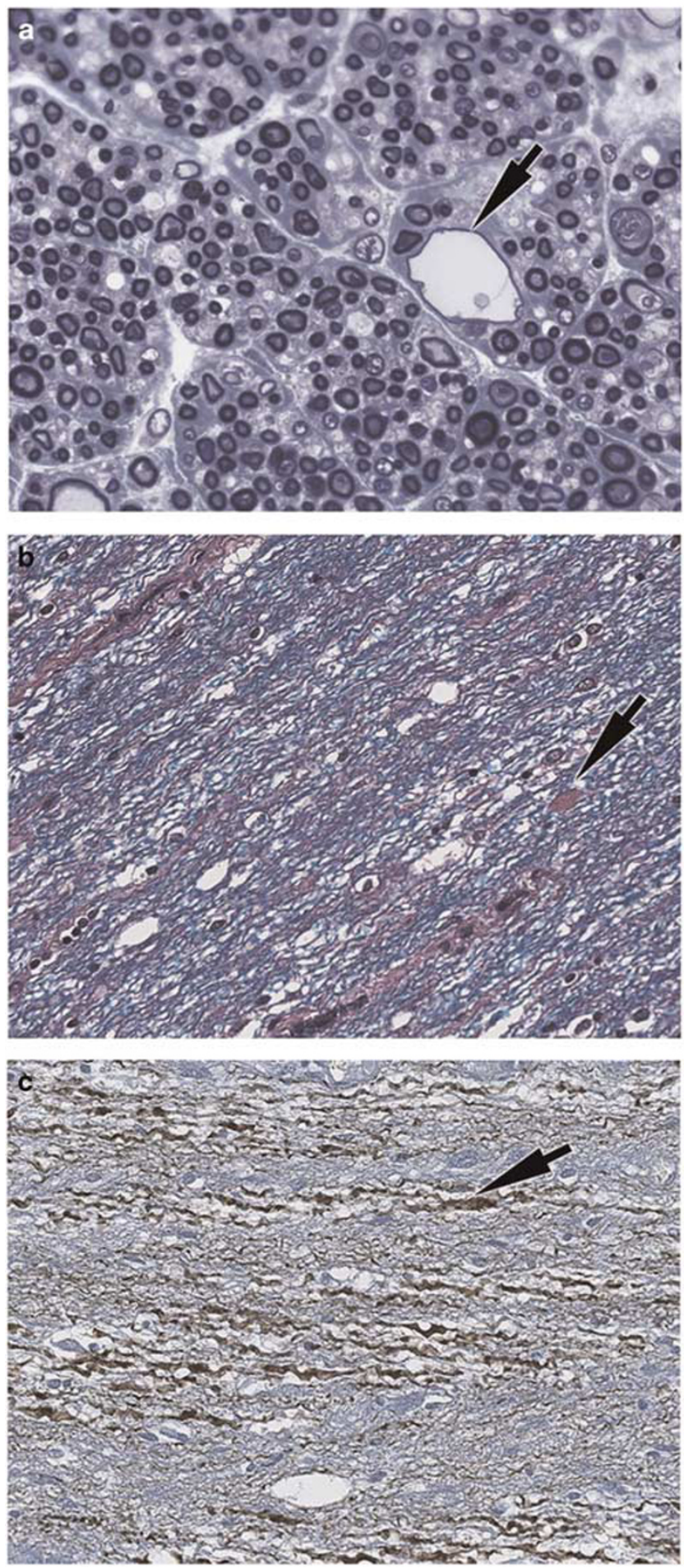

motor
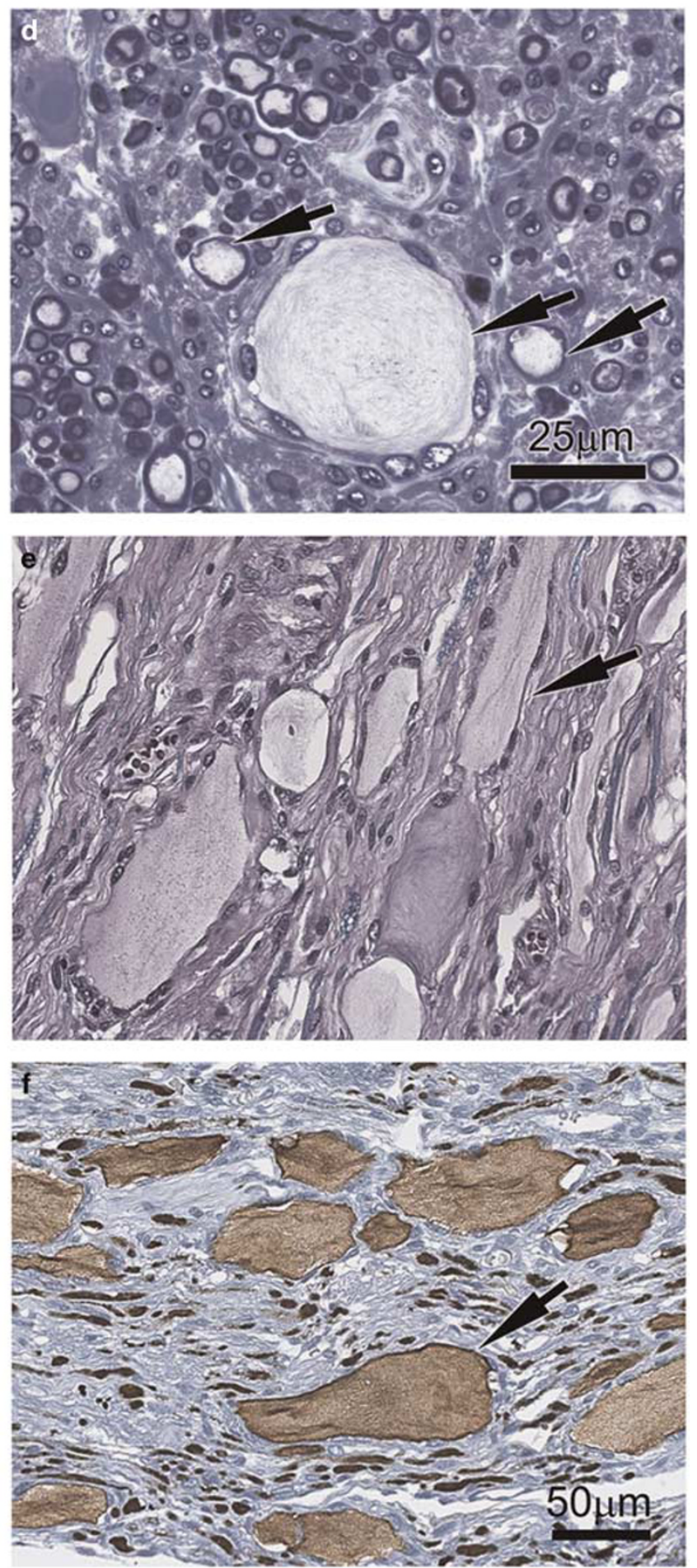

Figure 6 Severe axonal dilatation was observed in motor nerves. Panels show sensory nerves (a-c, case 3) and motor nerves (d-f, case 3). Epon sections of spinal sensory and motor rootlets stained with toluidine blue (a, d, e), respectively show larger and more abundant spheroids in motor nerves. Compared with (a) the sensory rootlet, (d) the motor rootlet shows far more axons with dilatation, as well as much more axonal loss. Longitudinal sections of the trigeminal sensory root $(\mathbf{b}, \mathbf{c})$ and the oculomotor nerve (e, f) stained with hematoxylin-eosin/luxol fast blue (b, e). Panels show more severe disease in the oculomotor nerve compared with the trigeminal nerve. Immunostaining with an antibody against neurofilament protein demonstrates much larger spheroids in the oculomotor nerve than in the trigeminal nerve (arrows in $\mathbf{c}, \mathbf{f}$ ), and that they are filled with neurofilaments. Axonal dilatation leads to myelin sheaths that are thin to absent (arrows in a, d, e). 

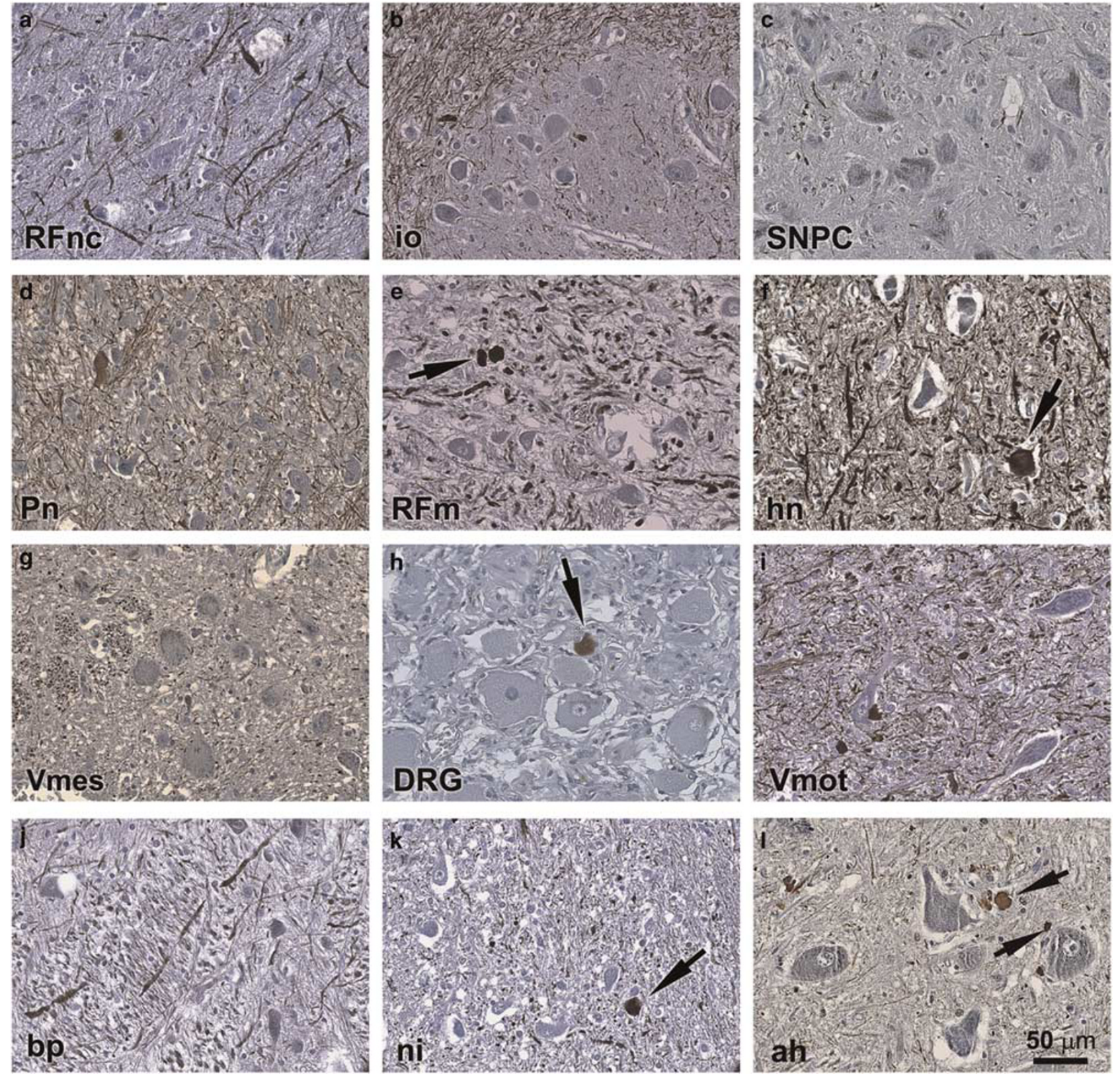

Figure 7 Central nervous system gray matter and ganglion neurons of HMSN/ACC patients do not display pathology. The reticular formation, nucleus cuneiformis (a, RFnc, case 5), inferior olive (b, io, case 7), substantia nigra pars compacta (c, SNPC, case 3), pontine nuclei (d, pn, case 8) reticular formation, medulla oblongata (e, RFm, case 4), hypoglossal nucleus (f, hn, case 8), trigeminal mesencephalic nucleus (g, Vmes, case 8), dorsal root ganglia (h, DRG, case 3), trigeminal motor nucleus (i, Vmot, case 5), basis pontis (j, bp, case 4), nucleus interpositus of the cerebellum (k, ni, case 4) and anterior horn (1, ah, case 7), all show axonal spheroids of varying sizes and in varying stages of degeneration when stained with an antibody against neurofilament protein. Despite these widespread axonal changes in HMSN/ACC patients, no neurons showed abnormalities and no neuronal death was seen in any nuclear, cortical or ganglionic structure. Arrows show axonal spheroids, including perisomatic axonal spheroids in central nervous system nuclei throughout central nervous system and peripheral ganglia (arrows in $\mathbf{l}, \mathbf{h}$, respectively).

KCC3 were shown in both sensory and motor neurons, ${ }^{17,18}$ studies in both animal models ${ }^{11}$ and human patients (this study) have revealed a greater pathology in motor than in sensory axons. This observation has been seen in many neurodegenerative disorders such as amyotrophic lateral sclerosis or lead neurotoxicity.
Spanning childhood and early adulthood, HMSN/ ACC is a neurodevelopmental as well as a neurodegenerative disorder caused by not only water influx in axons but also by deficits in KCC3-associated function during axons long projections of cortical neurons toward their distal targets. Axons fail to develop properly due to a KCC3 channelopathy, and 

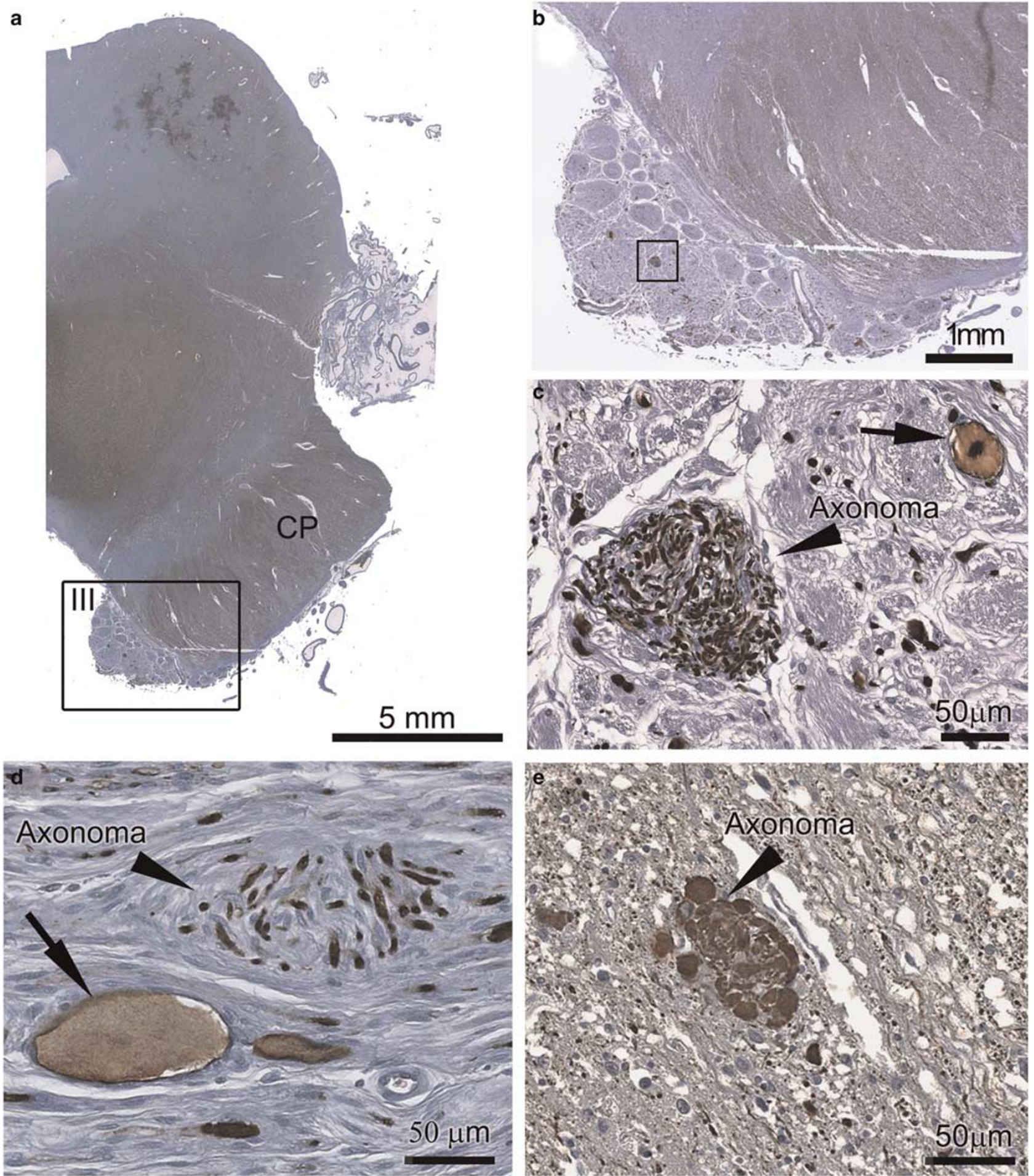

Figure 8 Tumor-like regenerative axon clusters termed axonomas were observed in both the central and peripheral nervous system. (a, case 4) Midbrain section at the level of the oculomotor nerve. The boxed area is shown in (b) and the exiting third nerve is seen to contain clusters of strongly neurofilament positive knots of tangled axonal overgrowth termed axonomas, one of which is shown (arrowhead in c). A dilated axon is also seen in the upper right, showing internal neurofilament accumulation at the center of the spheroid (arrow in c). The cauda equina in longitudinal section contained an enlarged axon (d, arrow, case 3) and adjacent axonoma (arrowhead). The spinal cord shows an axonoma (e, arrowhead, case 7) with a tendency to axonal dilatation seen at the periphery of the tumorlet, located in the dorsal horn. All sections were stained with an antibody against neurofilament protein. 

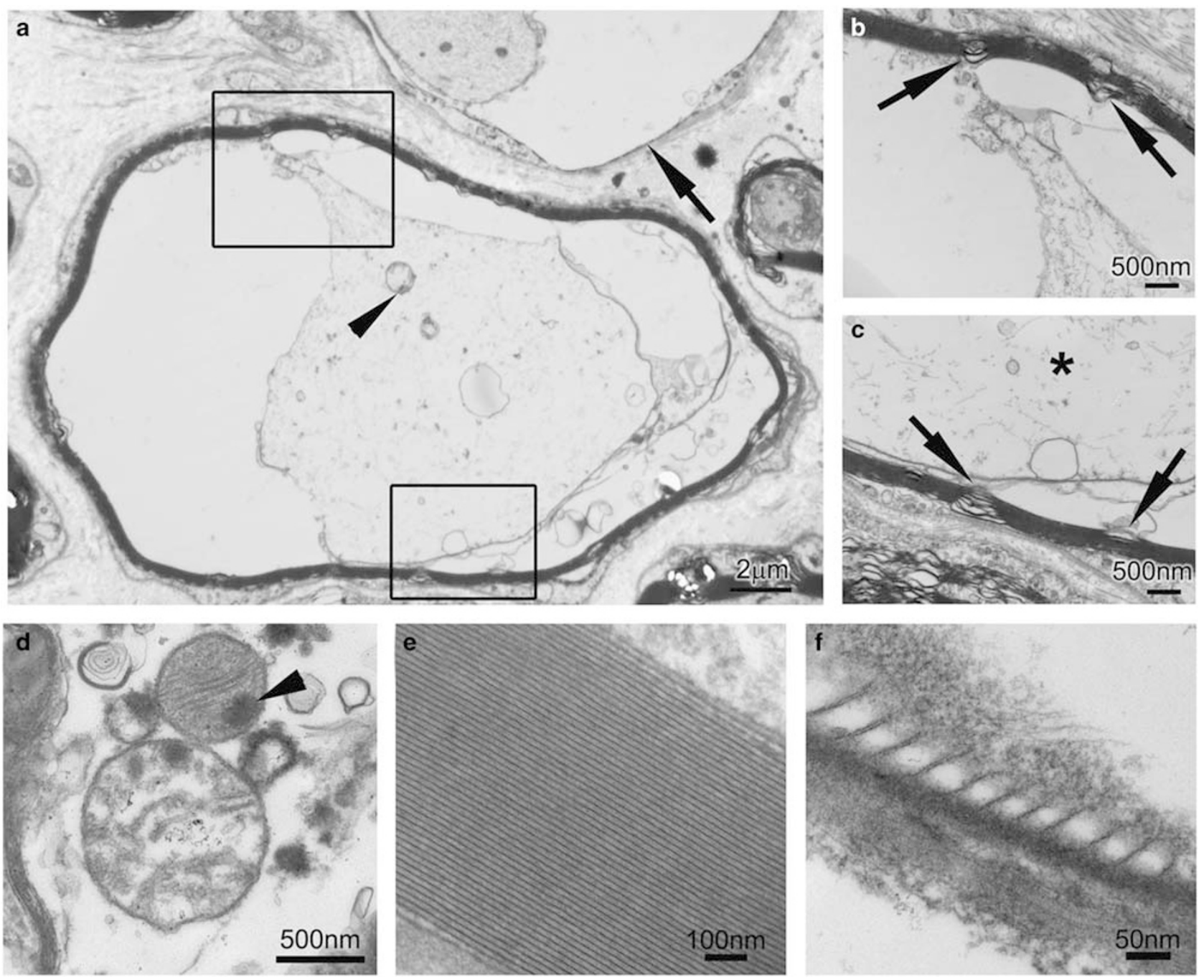

Figure 9 Electron microscopy of peripheral nerve from case 5 revealed axonal damage. (a) The third cranial nerve here shows two axonal spheroids. Myelin sheaths are attenuated to the point of being entirely absent in the upper axon (arrow). Circumferential dehiscence of the axoplasm is seen. Drawn boxes show minimal myelin sheath disintegration at points where the axoplasma is still adherent to the myelin (upper box in panel $\mathbf{b}$ and lower box in panel $\mathbf{c}$, arrows). There is also a dispersion of neurofilaments ( ${ }^{*}$ in $\mathbf{c}$ ). Flocculent densities are visible in remaining mitochondrion in the collapsed axoplasm (arrowhead in a) as well as in two mitochondria (arrowhead in d). (e) Myelin sheaths and (f) Schmidt-Lantermann clefts are anatomically well-preserved, despite the thin myelin, probably related to stretching of myelin around the enlarged axons.

subsequently degenerate in young adulthood, giving rise to both developmental and neurodegenerative aspects of the disease in a temporal continuum.

\section{Acknowledgments}

This work was supported by a Canadian Institutes of Health Research-Neuromuscular Research Partnership Program operating Grant (201671) to GAR and PAD, and a grant from the Fondation des Jumelles Coudé to GAR. Victoria Auer made the electron micrographs.

\section{Disclosure/conflict of interest}

The authors declare no conflict of interest.

\section{References}

1 Andermann E, Andermann F, Joubert M et al. Familial agenesis of the corpus callosum with anterior horn cell disease. A syndrome of mental retardation, areflexia and paraplegia. Trans Am Neurol Assoc 1972;97:242-244.

2 Filteau MJ, Pourcher E, Bouchard RH et al. Corpus callosum agenesis and psychosis in Andermann syndrome. Arch Neurol 1991;48:1275-1280.

3 Mathieu J, Bedard F, Prevost C et al. [Motor and sensory neuropathies with or without agenesis of the corpus callosum: a radiological study of 64 cases]. Can J Neurol Sci 1990;17:103-108.

4 Dupre N, Howard HC, Mathieu J et al. Hereditary motor and sensory neuropathy with agenesis of the corpus callosum. Ann Neurol 2003;54:9-18.

5 Larbrisseau A, Vanasse M, Brochu P et al. The Andermann syndrome: agenesis of the corpus callosum associated with 
mental retardation and progressive sensorimotor neuronopathy. Can J Neurol Sci 1984;11:257-261.

6 Howard HC, Mount DB, Rochefort D et al. The K-Cl cotransporter KCC3 is mutant in a severe peripheral neuropathy associated with agenesis of the corpus callosum. Nat Genet 2002;32:384-392.

7 Uyanik G, Elcioglu N, Penzien J et al. Novel truncating and missense mutations of the KCC3 gene associated with Andermann syndrome. Neurology 2006;66:1044-1048.

8 Salin-Cantegrel A, Riviere JB, Dupre N et al. Distal truncation of KCC3 in non-French Canadian HMSN/ ACC families. Neurology 2007;69:1350-1355.

9 Gamba G. Molecular physiology and pathophysiology of electroneutral cation-chloride cotransporters. Physiol Rev 2005;85:423-493.

10 Carpenter S. The pathology of Andermann syndromeCallosal Agenesis: A Natural Split Brain? Plenum: New York, NY, USA, 1994;27-30.

11 Shekarabi M, Moldrich RX, Rasheed S et al. Loss of neuronal potassium/chloride cotransporter 3 (KCC3) is responsible for the degenerative phenotype in a conditional mouse model of hereditary motor and sensory neuropathy associated with agenesis of the corpus callosum. J Neurosci 2012;32:3865-3876.

12 Sung JH. Tangled masses of central axons (central axonomas) in the brain stem: anatomical evidence for the regenerative growth of human central axons. J Neuropathol Exp Neurol 1987;46:200-213.

13 Lindwall C, Fothergill T, Richards LJ. Commissure formation in the mammalian forebrain. Curr Opin Neurobiol 2007;17:3-14.

14 Kamiguchi H, Hlavin ML, Yamasaki M et al. Adhesion molecules and inherited diseases of the human nervous system. Annu Rev Neurosci 1998;21:97-125.
15 Pearson MM, Lu J, Mount DB et al. Localization of the $\mathrm{K}(+)-\mathrm{Cl}(-)$ cotransporter, $\mathrm{KCC} 3$, in the central and peripheral nervous systems: expression in the choroid plexus, large neurons and white matter tracts. Neuroscience 2001;103:481-491.

16 Ding J, Delpire E. Deletion of KCC3 in parvalbumin neurons leads to locomotor deficit in a conditional mouse model of peripheral neuropathy associated with agenesis of the corpus callosum. Behav Brain Res 2014;274:128-136.

17 Shekarabi M, Salin-Cantegrel A, Laganiere J et al. Cellular expression of the $\mathrm{K}^{+}-\mathrm{Cl}^{-}$cotransporter KCC3 in the central nervous system of mouse. Brain Res 2011;1374:15-26.

18 Lucas O, Hilaire C, Delpire E et al. KCC3-dependent chloride extrusion in adult sensory neurons. Mol Cell Neurosci 2012;50:211-220.

This work is licensed under a Creative Commons Attribution-NonCommercialShareAlike 4.0 International License. The images or other third party material in this article are included in the article's Creative Commons license, unless indicated otherwise in the credit line; if the material is not included under the Creative Commons license, users will need to obtain permission from the license holder to reproduce the material. To view a copy of this license, visit http://creativecommons.org/licenses/ by-nc-sa/4.0/

(C) The Author(s) 2016

Supplementary Information accompanies the paper on Modern Pathology website (http://www.nature.com/ modpathol) 